Correspondence: Christian Soborg, Dept of Infectious Diseases, Hvidovre Hospital, Kettegårds alle 30, Hvidovre 2650, Denmark. E-mail: borg1@dadlnet.dk

Received: Sept 302013 | Accepted after revision: April 032014 | First published online: May 22014

Support statement: Copenhagen University Hospital (Hvidovre, Denmark) hold patents on the use of IP-10 as a marker for infection with Mycobacterium tuberculosis. P. Ravn and M. Ruhwald are registered as co-inventors. P.H. Andersen is employed by Statens Serum Institute which holds patent rights on antigens used in the QFT-IT test.

Conflict of interest: Disclosures can be found alongside the online version of this article at erj.ersjournals.com

Acknowledgements: We are grateful to Christina Thorstenisson (Dept of Infectious Disease, Copenhagen University Hospital, Hvidovre, Denmark) for statistical assistance and Bolette Søborg (Dept of Epidemiology Research, Statens Serum Institut, Copenhagen, Denmark) for enthusiastic commenting in the manuscript.

\title{
References
}

1 EPI-NEWS. Tuberculosis 2011, Part I. No. 50, 2012. www.ssi.dk/English/News/EPI-NEWS/2012/No\%2050\% 20-\%202012.aspx Date last updated: December 12, 2012. Date last accessed: January 3, 2014.

2 Taarnhoj GA, Engsig FN, Ravn P, et al. Incidence, risk factors and mortality of tuberculosis in Danish HIV patients 1995-2007. BMC Pulm Med 2011; 11: 26.

3 Horsburgh CR Jr. Priorities for the treatment of latent tuberculosis infection in the United States. N Engl J Med 2004; 350: 2060-2067.

4 Lawn SD, Bekker LG, Wood R. How effectively does HAART restore immune responses to Mycobacterium tuberculosis? Implications for tuberculosis control. AIDS 2005; 19: 1113-1124.

5 Pontali E, Pasticci MB, Matteelli A, et al. Tuberculosis and HIV co-infection: do we have a surveillance system in Europe? Eur Respir J 2011; 38: 1258-1260.

6 Podlekareva DN, Panteleev AM, Grint D, et al. Short- and long-term mortality and causes of death in HIV/ tuberculosis patients in Europe. Eur Respir J 2014; 43: 166-177.

7 Elzi L, Schlegel M, Weber R, et al. Reducing tuberculosis incidence by tuberculin skin testing, preventive treatment, and antiretroviral therapy in an area of low tuberculosis transmission. Clin Infect Dis 2007; 44: 94-102.

8 Whalen CC, Johnson JL, Okwera A, et al. A trial of three regimens to prevent tuberculosis in Ugandan adults infected with the human immunodeficiency virus. Uganda-Case Western Reserve University Research Collaboration. N Engl J Med 1997; 337: 801-808.

9 Durovni B, Saraceni V, Moulton LH, et al. Effect of improved tuberculosis screening and isoniazid preventive therapy on incidence of tuberculosis and death in patients with HIV in clinics in Rio de Janeiro, Brazil: a stepped wedge, cluster-randomised trial. Lancet Infect Dis 2013; 13: 852-858.

10 World Health Organisation. WHO policy on collaborative TB/HIV activities: guidelines for national programmes and other stake holders. Geneva, WHO, 2012.

11 Brock I, Ruhwald M, Lundgren B, et al. Latent tuberculosis in HIV positive, diagnosed by the M. tuberculosis specific interferon-gamma test. Respir Res 2006; 7: 56.

12 The Danish HIV Cohort Study. www.rigshospitalet.dk/RHenglish/Menu/Departments+and+Clinics/Finsen+Centre/ Department+of+Infectious+Diseases_old/Research/ Date last updated: November 13, 2013. Date last accessed: January 3, 2014.

13 Aichelburg MC, Rieger A, Breitenecker F, et al. Detection and prediction of active tuberculosis disease by a wholeblood interferon-gamma release assay in HIV-1-infected individuals. Clin Infect Dis 2009; 48: 954-962.

\section{Tuberculosis elimination: dream or reality? The case of Cyprus}

\author{
To the Editor:
}

The World Health Organization (WHO)-recommended strategy of tuberculosis (TB) control has evolved from the DOTS (directly observed treatment, short course) strategy to the Stop TB strategy [1, 2]. By breaking the chain of transmission through rapid diagnosis and effective treatment of infectious TB cases, this strategy allowed the development of national TB programmes and proved to be highly cost-effective $[3,4]$.

However, in order to accelerate the decline of the TB pandemic, WHO has planned a post-2015 strategy focusing on the concept of TB elimination [5-8]. To reach elimination (defined as fewer than one sputum smear-positive case per million inhabitants), countries should ensure that diagnosis and treatment of latent TB infection (LTBI) supplements detection and treatment of active TB disease [7-10].

A recent survey conducted by the European Respiratory Society (as part of its 2012-2013 Presidential plan) and WHO in Europe has demonstrated that the majority of countries do not have all the interventions 


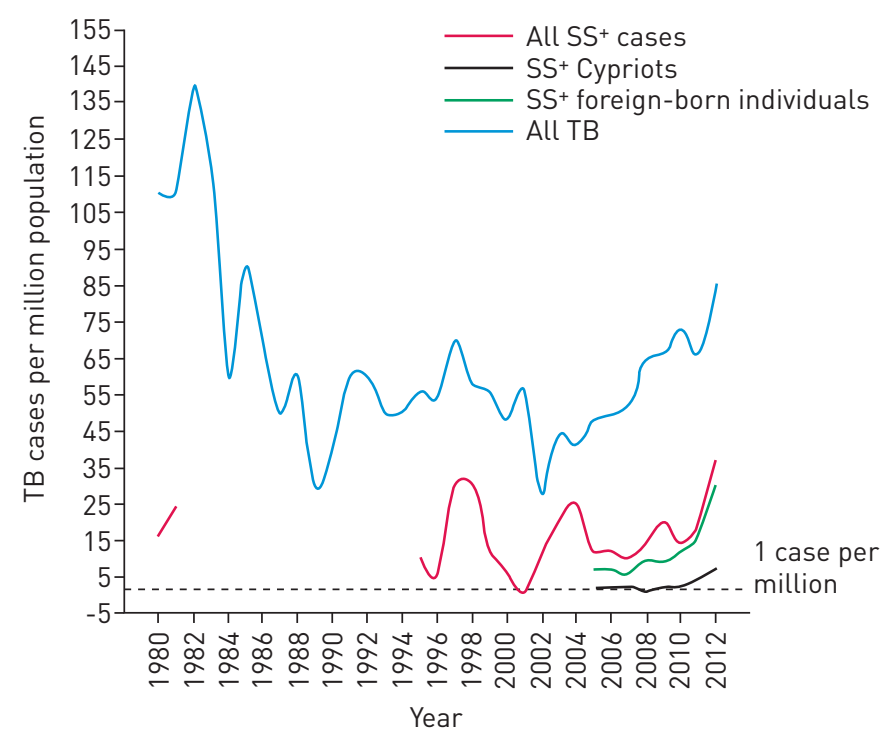

FIGURE 1 Cyprus tuberculosis (TB) cases per million population (19802012). $\mathrm{SS}^{+}$: sputum smear-positive.

in place to reach elimination [11], and stressed the importance of studying model countries able to demonstrate that $\mathrm{TB}$ elimination can be reached [12-14].

The aim of the present study was to show the progress achieved by Cyprus towards the elimination targets. Cyprus has been selected because of its solid TB control programme, the quality of surveillance data and health services, and its small population (865878 in 2012).

The way Cyprus covered the seven core areas considered essential to reach elimination (commitment, TB awareness and capacity of health systems, surveillance, quality laboratories and care, capacity to manage drugresistant TB and TB/HIV co-infection, introduction of new tools and operational research, and establishment of international collaborations) are described herein, and the impact indicators (notification of new sputum smear-positive case rates per million population) were analysed between 1995 and 2012 [7, 9].

Surveillance data were made available by the Ministry of Health of Cyprus in Nicosia from 1978 to 2012, based on European Union residents. Although data do not formally cover the whole of the island, Cyprus' health system offers its services to a large proportion of migrants entering from its northern occupied territory.

Cyprus has a solid TB control programme, and although an elimination plan has not been formalised, much has been done into this direction. Clinical [5] and laboratory [1] reference units have been identified, and civil society and scientific societies are officially collaborating with the national TB programme. Surveillance is regularly conducted including the necessary projection modelling to evaluate progress towards targets [11]. The national reference laboratory performs sputum smear microscopy, culture and drug susceptibility testing (DST) for first-line anti-TB drugs under external quality assurance by a supranational reference laboratory (Pasteur Institute, Athens, Greece), which is in charge of performing DST for second-line drugs. TB diagnostic and treatment services are available for free, while a nominal fee is charged for out-patients services (in 2011 out of 301 patients 255 (85\%) were outpatients). Anti-TB drugs are available and no stockout has ever been described. Multidrug-resistant TB cases are managed in a single reference centre, in isolation wards. Coordination with HIV/AIDS services has been established to ensure a one-stop service for diagnosis and treatment of TB/HIV co-infected individuals.

Screening for LTBI and contact screening are systematically performed as well as LTBI treatment (isoniazid for 6 or 9 months) although completion rates are not being monitored systematically. Impact indicators are summarised in figure 1.

The overall TB notification rate per million population decreased from 85 in 1980 to an historical minimum of 28 in 2002. Among infectious (sputum smear-positive) cases, the decline was from 24 to 11 per million.

In 2003, both the number and incidence of notified cases per million population reached a balance between individuals born in Cyprus and those born abroad. The proportion of cases among migrants to Cyprus has progressively increased, reaching a "plateau" > $80 \%$ in 2007. 
TB notifications and rates (all forms) declined progressively among natives from 31 in 1996 to six per million in 2004, then reaching a plateau around seven to eight per million. Among infectious cases, the reduction reached two cases per million in 2005, bordering the conventional elimination threshold of one per million. Among foreign-born individuals, the rates of notified cases instead increased to reach 54 overall and nine per million (sputum smear-positive) in 2009.

The analysis of TB trends in Cyprus shows that, under sound control measures, the number of cases had an annual average $9.4 \%$ decrease from 1997 to 2002 (from 46 to 20 cases), followed by a mild increase related to contribution of Mycobacterium tuberculosis strains from the immigrant population.

Cyprus health services attract more and more foreign-born cases from the Turkish-occupied area of the island [15].

Out of 244 migrants with TB diagnosed between 2007 and 2012, 89 (36.50\%) were born in Asia, 91 $(37.30 \%)$ in eastern Europe, 33 (13.50\%) in former Soviet Union countries, 22 (9.00\%) in west Europe and nine (3.70\%) in Africa. In terms of essential control interventions, Cyprus has developed efficient casefinding (estimated case-detection rate 100\% in 2012 and $>87 \%$ since 1990, diagnostic delay $2-4$ days) and treatment services (case success rate $>85 \%$ since 2006 ).

This is further testified by the low prevalence of multidrug resistance $(4.4 \%$ among new cases and retreatment cases together; 10 cases diagnosed since 2005).

Therefore, Cyprus has virtually reached the elimination phase in its native born population (fig. 1). Unfortunately, it is difficult to maintain such a low level of $M$. tuberculosis transmission in the population; even a few TB cases deriving from the continuous flow of immigrants are, at the moment, sufficient to prevent further progress.

Thus, several interventions need to be improved to pursue elimination in this country. First of all, focusing on at-risk populations, and especially migrants, is essential: innovative interventions of early and active case detection must be strengthened, accompanied by preventive treatment for persons at high risk. In addition, the Cyprus government needs to implement other measures, including: development of specific targets and plans for TB elimination at the national level accompanied by a well-thought projection model that assesses the potential impact of different measures, including all current, and potential future, new diagnostics and drugs; introduction of rapid molecular diagnostics (a few Xpert MTB/RIF (Cepheid, Sunnyvale, CA, USA) machines could easily cover the entire population of Cyprus) for both native and migrant populations seeking care; surveillance using systematic fingerprinting of all the strains isolated to better identify and contain microepidemics; and implementation of electronic surveillance and mechanisms to ensure validation and quality control of data, and improvement of the existing LTBI register (to allow evaluation of LTBI treatment completion rates). Finally, universal health coverage that translates into easy access to highquality diagnosis and treatment without catastrophic expenditures, and social protection mechanisms covering for income losses among people with TB during their months-long treatment need also to be introduced to protect the most vulnerable individuals.

The Cyprus model confirms that targeting elimination is feasible although maintaining the TB epidemic below this threshold requires continuous commitment and funding. Among additional core policies and interventions needed at the national level to accelerate incidence decline, those related to diagnosis and treatment of LTBI are the most important [16].

O @ERSpublications

Cyprus achieved TB elimination by comprehensive effort but imported cases do not allow it to stay below the threshold http://ow.ly/uWiDB

Constantia Voniatis ${ }^{1,6}$, Giovanni Battista Migliori ${ }^{2,6}$, Michael Voniatis ${ }^{3}$, Andreas Georgiou ${ }^{4}$, Lia D’Ambrosio ${ }^{2}$, Rosella Centis ${ }^{2}$ and Mario C. Raviglione ${ }^{5}$

${ }^{1}$ Ministry of Health, Nicosia, Cyprus. ${ }^{2}$ WHO Collaborating Centre for TB and Lung Diseases, Fondazione S. Maugeri, Care and Research Institute, Tradate, Italy. ${ }^{3}$ Cyprus Foundation of Immunology and Allergology, Nicosia, Cyprus.

${ }^{4}$ Tuberculosis Reference Centre, Pulmonology Clinic, Nicosia General Hospital, Nicosia, Cyprus. ${ }^{5}$ Global TB Programme, World Health Organization, Geneva, Switzerland. ${ }^{6}$ These authors contributed equally.

Correspondence: Giovanni Battista Migliori, WHO Collaborating Centre for Tuberculosis and Lung Diseases, Fondazione Salvatore Maugeri, Care and Research Institute, via Roncaccio 16, 21049, Tradate (VA), Italy.

E-mail: giovannibattista.migliori@fsm.it

Received: March 072014 | Accepted after revision: March 192014 | First published online: April 172014

Conflict of interest: None declared. 


\section{References}

1 Sotgiu G, Spanevello A, Migliori GB. History of tuberculosis and drug resistance. N Engl J Med 2013; 368: 88-89.

2 Raviglione M, Marais B, Floyd K, et al. Scaling up interventions to achieve global tuberculosis control: progress and new developments. Lancet 2012; 379: 1902-1913.

3 Migliori GB, Zellweger JP, Abubakar I, et al. European union standards for tuberculosis care. Eur Respir J 2012; 39: 807-819.

4 Migliori GB, Sotgiu G, Blasi F, et al. Towards the development of EU/EEA Standards for Tuberculosis Care (ESTC). Eur Respir J 2011; 38: 493-495.

5 Broekmans JF, Migliori GB, Rieder HL, et al. European framework for tuberculosis control and elimination in countries with a low incidence. Eur Respir J 2002; 19: 765-775.

6 Veen J, Migliori GB, Raviglione M, et al. Harmonisation of TB control in the WHO European region: the history of the Wolfheze Workshops. Eur Respir J 2011; 37: 950-959.

7 Diel R, Loddenkemper R, Zellweger J-P, et al. Old ideas to innovate TB control: preventive treatment to achieve elimination. Eur Respir J 2013; 42: 785-801.

8 World Health Organization. Report of the 13th Meeting WHO Strategic and Technical Advisory Group for Tuberculosis (STAG-TB) 11-12 June 2013. Document WHO/HTM/TB/2013.09. Geneva, World Health Organization, 2013.

9 European Centre for Disease Prevention and Control. Framework Action Plan to fight tuberculosis in the European Union. Stockholm, 2008. http://ecdc.europa.eu/en/publications/publications/0803_spr_tb_action_plan.pdf

10 Clancy L, Rieder HL, Enarson DA, et al. Tuberculosis elimination in the countries of Europe and other industrialized countries. Eur Respir J 1991; 4: 1288-1295.

11 D’Ambrosio L, Dara M, Tadolini M, et al. Tuberculosis elimination: theory and practice in Europe. Eur Respir J 2014; 43: 1410-1420.

12 Blasi F, Barnes PJ, Gaga M, et al. Future directions for the ERS: Presidential plans. Eur Respir J 2013; 42: 875-880.

13 Blasi F, Reichman LB, Migliori GB. Presenting the European Forum for TB Innovation: innovative thinking in progressing towards TB elimination in Europe. Eur Respir J 2012; 40: 806-808.

14 Blasi F, Dara M, van der Werf MJ, et al. Supporting TB clinicians managing difficult cases: the ERS/WHO Consilium. Eur Respir J 2013; 41: 491-494.

15 Cyprus Ministry of Health. Annual health report, Nicosia; 2010. Available from: http://www.moh.gov.cy/moh/moh. nsf/page09_gr/page09_gr?OpenDocument Date last accessed: March 28, 2014.

16 Borgdorff MW, van den Hof S, Kremer K, et al. Progress towards tuberculosis elimination: secular trend, immigration and transmission. Eur Respir J 2010; 36: 339-347. 